\title{
Transformée en paquets d'ondelettes des signaux stationnaires: comportement asymptotique des densités spectrales
}

Loïc Hervé

Résumé. On considère la transformée en paquets d'ondelettes associée à un filtre polynomial QMF. Soit $X=\left\{X_{n}\right\}_{n \in \mathbb{Z}}$ un signal aléatoire stationnaire à densité spectrale $f$ continue. On démontre que les $2^{n}$ signaux, générés à partir de $X$ après $n$ itérations de la transformée, "convergent" vers des bruits blancs quand $n \rightarrow+\infty$. Si $f$ est hölderienne, la vitesse de convergence est exponentielle.

Abstract. We consider quadrature mirror filters, and the associated wavelet packet transform. Let $X=\left\{X_{n}\right\}_{n \in \mathbb{Z}}$ be a stationary signal which has a continuous spectral density, $f$. We prove that the $2^{n}$ signals, obtained from $X$ by $n$ iterations of the transform, "converge" to white noises when $n \rightarrow+\infty$. If $f$ is holderian, the convergence rate is exponential. 


\section{Enoncé des résultats.}

On désigne par $\left(E,\|\cdot\|_{\infty}\right)$ l'espace des fonctions continues 1-périodiques muni de la norme uniforme, et pour $\eta \in] 0,1]$, on note $\left(E^{\eta},\|\cdot\|_{\eta}\right)$ le sous-espace des fonctions uniformément $\eta$-hölderiennes, avec

$$
\|f\|_{\eta}=\|f\|_{\infty}+m_{\eta}(f),
$$

où

$$
m_{\eta}(f)=\sup \left\{\frac{|f(y)-f(x)|}{|y-x|^{\eta}}, x \neq y\right\} .
$$

Soit $H_{0}(\lambda)=\sum_{k=0}^{N} h_{k}^{0} e^{2 i \pi k \lambda}$ un polynôme trigonométrique tel que $H_{0}(0)=1$ et

$$
\left|H_{0}\left(\frac{\lambda}{2}\right)\right|^{2}+\left|H_{0}\left(\frac{\lambda}{2}+\frac{1}{2}\right)\right|^{2}=1, \text { pour tout } \lambda \in[0,1] .
$$

On suppose que les $h_{k}^{0}$ sont des nombres réels, et on définit $h_{k}^{1}=$ $(-1)^{k+1} h_{-1-k}^{0}$ pour $k=-N-1, \ldots,-1$, et

$$
H_{1}(\lambda)=\sum_{k=-N-1}^{-1} h_{k}^{1} e^{2 i \pi k \lambda}=e^{-2 i \pi \lambda} \overline{H_{0}\left(\lambda+\frac{1}{2}\right)} .
$$

Notons que $H_{0}(0)=H_{0}(1)=H_{1}(1 / 2)=1, H_{1}(0)=H_{1}(1)=H_{0}(1 / 2)$ $=0$, et que $H_{1}$ vérifie également la relation (1). Un tel couple $\left(H_{0}, H_{1}\right)$ est appelé QMF (quadrature mirror filters) [3], [8]. La méthode d'analyse spectrale des signaux aléatoires, développée dans [2], consiste à itérer, à partir d'un processus aléatoire initial $X=\left(X_{n}\right)_{n \in \mathbb{Z}}$, les deux opérations de filtrage $T_{0}$ et $T_{1}$ définies par

$$
\left(T_{j} X\right)_{n}=\sqrt{2} \sum_{k \in \mathbb{Z}} h_{k}^{j} X_{2 n-k}, \quad j=0,1, \quad n \in \mathbb{Z},
$$

où par convention $h_{k}^{0}=0$ si $k \notin[0, N]$, et $h_{k}^{1}=0$ si $k \notin[-N-1,-1]$.

Cet algorithme, qui en d'autres termes effectue la transformée en paquets d'ondelettes de $X$ [1], fournit une famille arborescente de signaux stationnaires: après $n$ itérations, on dispose des $2^{n}$ processus $T_{\omega_{n}} \cdots T_{\omega_{1}} X$, où les $\omega_{i}$ décrivent $\{0,1\}$.

On note $\Omega=\{0,1\}^{\mathbb{N}^{*}}, u_{0}=\left|H_{0}\right|^{2}, u_{1}=\left|H_{1}\right|^{2}$, et $P_{0}, P_{1}$ les opérateurs de transition définis sur $E$ par

$$
P_{j} f(\lambda)=u_{j}\left(\frac{\lambda}{2}\right) f\left(\frac{\lambda}{2}\right)+u_{j}\left(\frac{\lambda}{2}+\frac{1}{2}\right) f\left(\frac{\lambda}{2}+\frac{1}{2}\right),
$$


où $\lambda \in[0,1], j=0,1$. On démontre dans [2] le résultat suivant

\section{Théorème 1.1 .}

i) Si $X=\left(X_{n}\right)_{n \in \mathbb{Z}}$ est un processus stationnaire $d u$ second ordre, les processus $T_{0} X$ et $T_{1} X$ sont également stationnaires. Si $X$ a une densité spectrale $f$, alors $P_{0} f$ et $P_{1} f$ sont les densités spectrales respectivement de $T_{0} X$ et $T_{1} X$, et plus généralement chaque processus $T_{\omega_{n}} \cdots T_{\omega_{1}} X$ admet une densité spectrale égale à $P_{\omega_{n}} \cdots P_{\omega_{1}} f$.

ii) Soit $Q_{0}$ le nombre de zéros de $H_{0}$. On suppose que, pour tout $p \in\left\{1, \ldots, Q_{0}\right\}$, pour tout $k \in\left\{1, \ldots, 2^{p}-2\right\}$, il existe $\ell \in\{0, \ldots, p\}$, tel que

$$
H_{0}\left(\frac{k 2^{\ell}}{2^{p}-1}+\frac{1}{2}\right) \neq 0 .
$$

Si $f \in E$, alors, pour presque tout $\omega=\left(\omega_{1}, \omega_{2}, \ldots, \omega_{n}, \ldots\right) \in \Omega($ au sens de la probabilité produit équiprobable sur $\Omega)$, la suite de processus $\left(T_{\omega_{n}} \cdots T_{\omega_{1}} X\right)_{n \geq 1}$ "converge" vers un bruit blanc, c'est-à-dire $\left(P_{\omega_{n}} \cdots\right.$ $\left.P_{\omega_{1}} f\right)_{n \geq 1}$ converge vers une constante $c(f, \omega)$ quand $n \rightarrow+\infty$.

Le point i) résulte d'un calcul élémentaire, et l'assertion ii) du Théorème de Ionescu-Tulcea et Marinescu [6] et de la Loi des grands nombres. Signalons que la condition (3) est exactement l'hypothèse sur les cycles périodiques invariants donnée dans [2]. Dans ce travail, sous une hypothèse du même type mais un peu plus forte que (3), nous nous proposons, d'une part de généraliser la propriété de convergence du ii) à tout $\omega \in \Omega$, et d'autre part de prouver que la vitesse de convergence est exponentielle quand $f$ est hölderienne.

Théorème 1.2. Soit $Q$ le nombre de zéros de $H_{0} H_{1}$. On suppose que, pour tout $p \in\{1, \ldots, Q\}$, pour tout $k \in\left\{1, \ldots, 2^{p}-2\right\}$, il existe $\ell \in\{0, \ldots, p\}$, tel que

$$
H_{0}\left(\frac{k 2^{\ell}}{2^{p}-1}\right) H_{1}\left(\frac{k 2^{\ell}}{2^{p}-1}\right) \neq 0 .
$$

Soit $X$ un processus stationnaire du second ordre admettant une densité spectrale $f$ continue, et soit $\omega=\left(\omega_{1}, \ldots, \omega_{n}, \ldots\right) \in \Omega$ quelconque. Alors la mesure spectrale $P_{\omega_{n}} \cdots P_{\omega_{1}} f d u$ processus $T_{\omega_{n}} \cdots T_{\omega_{1}} X$ converge uniformément vers une constante $c(f, \omega)$ quand $n \rightarrow+\infty$, c'est$\grave{a}$-dire,

$$
\lim _{n \rightarrow+\infty}\left\|P_{\omega_{n}} \cdots P_{\omega_{1}} f-c(f, \omega)\right\|_{\infty}=0
$$


Théorème 1.3. On conserve les hypothèses et notations du théorème précédent. Si $f \in E^{\eta}$, alors il existe deux constantes $D>0$ et $\rho \in[0,1[$ (indépendantes de $f$ ) telles que l'on ait, pour tout $\omega=\left(\omega_{1}, \ldots, \omega_{n}, \ldots\right)$ $\in \Omega$,

$$
\left\|P_{\omega_{n}} \cdots P_{\omega_{1}} f-c(f, \omega)\right\|_{\eta} \leq D \rho^{n}\|f\|_{\eta}
$$

REMARques. a) La propriété (6) montre que la suite d'opérateurs $\left(P_{\omega_{n}} \cdots P_{\omega_{1}}\right)_{n \geq 1}$ converge en norme dans $E^{\eta}$ avec une vitesse exponentielle. L'étude du comportement asymptotique des spectres discrets dans l'arbre de filtrage, que nous n'abordons pas ici, a été traitée dans [2]. Par ailleurs une étude plus précise des itérées de $T_{0}$ a été faite dans [5] dans le cadre des filtres polynomiaux non nécessairement QMF.

b) L'hypothèse (3) est une condition nécessaire et suffisante pour que $H_{0}$ engendre une analyse multirésolution, et elle assure que la suite $\left\{P_{0}^{n} f, n \geq 1\right\}$ converge uniformément vers $f(0)$ pour tout $f \in E$, voir [2]. Pour $\omega \in \Omega$, on note $\mu_{\omega}$ la mesure de probabilité sur le tore définie par

$$
\int f d \mu_{\omega}=c(f, \omega), \quad f \in E .
$$

Si $\omega=(0,0, \ldots)$, le résultat ci-dessus montre que $\mu_{\omega}=\delta_{0}$ (masse de Dirac en 0$)$. Plus généralement, si $\omega$ est de la forme $\omega=\left(\omega_{1}, \ldots, \omega_{r}, 0\right.$, $0, \ldots)$, on a $\mu_{\omega}=\sum_{k=0}^{2^{r}-1} u_{\omega_{r}}(k / 2) \cdots u_{\omega_{1}}\left(k / 2^{r}\right) \delta_{\left(k /\left(2^{r}\right)\right)}$.

Cependant ce type de propriété ne s'étend pas à tous les éléments de $\Omega$. Plus précisément, soient $\nu$ la mesure produit équiprobable sur $\Omega, \mathcal{D}$ l'ensemble des points dyadiques de $[0,1[$, et soit enfin $A$ le sousensemble de $\Omega$ formé des $\omega$ tels que $\mu_{w}$ soit de la forme

$$
\mu_{\omega}=\sum_{a \in \mathcal{D}} \alpha_{a}(\omega) \delta_{a}
$$

où les $\alpha_{a}(\omega)$ sont des réels positifs tels que $\sum_{a \in \mathcal{D}} \alpha_{a}(\omega)=1$. Alors $\nu(A)=0$. En effet, on établit aisément par récurrence que

$$
\int_{0}^{1} f(\lambda) d \lambda=2^{-n} \sum_{\omega_{1}, \ldots, \omega_{n} \in\{0,1\}} \int_{0}^{1} P_{\omega_{n}} \cdots P_{\omega_{1}} f(\lambda) d \lambda,
$$

pour tout $f \in E$ et pour tout $n \geq 1$. D'où, grâce au théorème de convergence dominée sur $(\Omega, \nu)$,

$$
\int_{0}^{1} f(\lambda) d \lambda=\int_{\Omega} c(f, \omega) d \nu(\omega) .
$$


Donc, pour tout borélien $B$, on a $m(B)=\int_{\Omega} \mu_{\omega}(B) d \nu(\omega)$, où $m$ est la mesure de Lebesgue sur $[0,1]$. Pour $B=\mathcal{D}$, on obtient $0=m(\mathcal{D}) \geq$ $\nu(A)$. Donc $\nu(A)=0$.

Signalons également que, si $\omega=(1,1, \ldots)$, alors $\mu_{\omega}$ est une mesure continue: $\mu_{\omega}(\{x\})=0$ pour tout $x \in[0,1[$. En effet supposons que $\mu_{\omega}(\{x\})>0$. On sait que $\mu_{\omega}$ est invariante sous l'action de $P_{1}$ et $\Delta$, où $\Delta$ est la transformation définie par $\Delta \lambda=2 \lambda$ (modulo1), voir [7]. On en déduit aisément que $x$ est nécessairement un point fixe pour une certaine puissance $\Delta^{p}$ de $\Delta$, et que $u_{1}\left(\Delta^{k} x\right)=1$ pour $k=1, \ldots, p$, ce qui contredit l'hypothèse (4). Donc $\mu_{\omega}(\{x\})=0$. De même, si $\omega=(0,1,0,1, \ldots)$, ou plus généralement si $\omega$ est cyclique, on peut montrer que $\mu_{\omega}$ est une mesure continue.

c) Pour tout $n \geq 1$, l'application $A_{n}$ définie par

$$
f \longmapsto\left(P_{\omega_{n}} \cdots P_{\omega_{1}} f\right)_{\omega_{1}, \ldots, \omega_{n} \in\{0,1\}}
$$

est injective de $E$ dans $E^{2^{n}}$. Il suffit de le vérifier pour $n=1$. $\theta_{r}$, pour $\lambda$ fixé, on a ${ }^{t}\left[P_{0} f(2 \lambda), P_{1} f(2 \lambda)\right]=\mathcal{A}(\lambda){ }^{t}[f(\lambda), f(\lambda+1 / 2)]$, où $\mathcal{A}(\lambda)$ est une matrice carrée d'ordre 2 qui s'exprime aisément à l'aide de $u_{0}(\lambda)$ et $u_{1}(\lambda)$, et dont le déterminant est $D(\lambda)=\left(u_{0}(\lambda)\right)^{2}-\left(u_{1}(\lambda)\right)^{2}$. L'injectivité de $A_{1}$ résulte du fait que $D$ a un nombre fini de zéros.

Par conséquent si deux processus $X$ et $Y$ admettent des densités spectrales $f_{X}$ et $f_{Y}$ distinctes, alors $A_{n} f_{X} \neq A_{n} f_{Y}$ pour tout $n \geq 1$, et en ce sens, la transformée en ondelettes des signaux stationnaires fournit un procédé d'analyse spectrale. Cependant, la propriété d'injectivité de $A_{n}$ peut se "dégrader" quand $n \rightarrow+\infty$. Plus précisément, considérons l'exemple du filtre de Haar, $H_{0}(\lambda)=\left(1+e^{2 i \pi \lambda}\right) / 2$. Un calcul simple montre que, pour $f(\lambda)=\sin 2 \pi \lambda$, on a $P_{0} f=f / 2$ et $P_{1} f=-f / 2$, de sorte que $c(f, \omega)=0$ pour tout $\omega \in \Omega$. En d'autres termes, l'application $f \mapsto c(f, \cdot)$ associée au filtre de Haar n'est pas injective. Nous ne sommes pas parvenus à étudier, pour $H_{0}$ quelconque, l'injectivité de $f \mapsto c(f, \cdot)$.

La suite de ce papier est consacrée à la démonstration des théorèmes 1.2 et 1.3 qui repose, d'une part sur la positivité des opérateurs $P_{0}, P_{1}$ et la notion de points périodiques [2], [4] (étude dans $E$ ), et d'autre part sur le Théorème de Ionescu-Tulcea et Marinescu et des arguments de compacité (étude dans $E^{\eta}$ ). 


\section{Démonstration du Théorème $\mathbf{1 . 2}$.}

Dans ce paragraphe, nous nous proposons de démontrer le Théorème 1.2. Pour simplifier, on notera, pour tout $n \in \mathbb{N}^{*}$ et tout $\omega=$ $\left(\omega_{1}, \ldots, \omega_{n}, \ldots\right) \in \Omega$

$$
\Pi_{n}^{\omega} f(\lambda)=P_{\omega_{n}} \cdots P_{\omega_{1}} f(\lambda), \quad f \in E, \lambda \in[0,1]
$$

Rappelons que $u_{1}(\lambda)=u_{0}(\lambda+1 / 2)$. Les opérateurs $P_{0}$ et $P_{1}$ définis par (2) sont bornés, positifs sur $E$, et vérifient $P_{0} 1=P_{1} 1=1$, où 1 est la fonction identiquement égale à 1 . De même chaque opérateur $\Pi_{n}^{\omega}$ est positif, borné sur $E$, et vérifie

$$
\Pi_{n}^{\omega} 1=1, \quad\left\|\Pi_{n}^{\omega} f\right\|_{\infty} \leq\|f\|_{\infty}, \quad \text { pour tout } f \in E
$$

On définit, pour $k \in \mathbb{N}^{*}, \mathcal{T}_{k}=\operatorname{vect}\left\{e^{-2 i \pi(k+1) \lambda}, \ldots, e^{2 i \pi(k+1) \lambda}\right\}$. Notons que $u_{0}$ et $u_{1}$ appartiennent à $\mathcal{T}_{N}$. Pour de simples raisons de degré, il est clair que, si $f$ est un polynôme trigonométrique, alors les fonctions $\Pi_{n}^{\omega} f$ appartiennent à $\mathcal{T}_{N}$ pour $n$ assez grand. De même, on montre facilement que $P_{0}, P_{1}$, et donc $\Pi_{n}^{\omega}$, opèrent sur $\mathcal{T}_{N}$.

Soient $S_{0}$ et $S_{1}$ les applications définies par

$$
S_{j} \lambda=\frac{\lambda+j}{2}, \quad \lambda \in[0,1], j=0,1
$$

Pour $\lambda \in[0,1], \omega \in \Omega$, et $n \in \mathbb{N}^{*}$, on note $T_{n, \lambda}^{\omega}$ l'ensemble des points $\sigma_{n} \cdots \sigma_{1} \lambda$ tels que $\sigma_{i} \in\left\{S_{0}, S_{1}\right\}$ et $u_{\omega_{n}}\left(\sigma_{1} \lambda\right) \cdots u_{\omega_{1}}\left(\sigma_{n} \cdots \sigma_{1} \lambda\right)>0$. Compte-tenu des identités $u_{j}(\lambda / 2)+u_{j}(\lambda / 2+1 / 2)=1$, l'ensemble $T_{n, \lambda}^{\omega}$ n'est jamais vide. On notera $[a]$ la partie entière d'un réel $a$, et $\theta$ le shift défini sur $\Omega$ par

$$
\theta \omega=\theta\left(\omega_{1}, \ldots, \omega_{n}, \ldots\right)=\left(\omega_{2}, \ldots, \omega_{n}, \ldots\right) .
$$

La démonstration du Théorème 1.2 utilise les deux lemmes techniques suivants:

Lemme 2.1. Soit $h$ une fonction de $E$ à valeurs positives ou nulles, et soient $\omega \in \Omega, \lambda \in[0,1], m, \ell \in \mathbb{N}^{*}$, et enfin $y=\tau_{\ell} \cdots \tau_{1} \lambda \in T_{\ell, \lambda}^{\theta^{m} \omega}$. Si $\Pi_{m}^{\omega} h(y)>0$, alors on a $\Pi_{\ell+m}^{\omega} h(\lambda)>0$. 
Lemme 2.2. On note $r=\left[\log _{2}(2 N+1)\right]+1+2 Q$. Soit $h$ une fonction de $\mathcal{T}_{N}$ à valeurs positives ou nulles, mais non identiquement nulle. Il existe $\delta>0$ telle que

$$
\begin{gathered}
P_{\mu_{n}} \cdots P_{\mu_{1}} P_{1} P_{\omega_{r}} \cdots P_{\omega_{1}} h(\lambda) \geq \delta, \quad \text { pour tous } \lambda \in[0,1], n \geq 1, \\
\omega_{i}, \mu_{i} \in\{0,1\} .
\end{gathered}
$$

Preuve Du Lemme 2.1. On montre aisément par récurrence que

$$
\Pi_{m}^{\omega} h(\lambda)=\sum_{\sigma_{1}, \ldots, \sigma_{m} \in\left\{S_{0}, S_{1}\right\}} u_{\omega_{m}}\left(\sigma_{1} \lambda\right) \cdots u_{\omega_{1}}\left(\sigma_{m} \cdots \sigma_{1} \lambda\right) h\left(\sigma_{m} \cdots \sigma_{1} \lambda\right)
$$

Comme $y \in T_{\ell, \lambda}^{\theta^{m} \omega}$, on a $A=u_{\omega_{m+\ell}}\left(\tau_{1} \lambda\right) \cdots u_{\omega_{m+1}}\left(\tau_{\ell} \cdots \tau_{1} \lambda\right)>0$. Le Lemme 2.1 se déduit alors de l'inégalité

$$
\begin{aligned}
& \Pi_{m+\ell}^{\omega} h(\lambda) \\
& \quad \geq \sum_{\sigma_{\ell+1}, \ldots, \sigma_{\ell+m} \in\left\{S_{0}, S_{1}\right\}}^{A} u_{\omega_{m}}\left(\sigma_{\ell+1} y\right) \cdots u_{\omega_{1}}\left(\sigma_{\ell+m} \cdots \sigma_{\ell+1} y\right) h\left(\sigma_{\ell+m} \cdots \sigma_{\ell+1} y\right) \\
& =A \Pi_{m}^{\omega} h(y)
\end{aligned}
$$

Avant de donner la preuve du Lemme 2.2, commençons par faire quelques rappels sur la notion de points périodiques [2], [4], et le lien avec la condition (4). Pour $p \in \mathbb{N}^{*}$, on dit que $\lambda \in[0,1]$ est un point $p$-périodique s'il existe $p$ éléments $\sigma_{1}, \ldots, \sigma_{p}$ de $\left\{S_{0}, S_{1}\right\}$ tels que $\sigma_{p} \cdots \sigma_{1} \lambda=\lambda$, et si $p$ est le plus petit entier pour lequel on a une telle relation (de manière équivalente, si $\Delta^{p} \lambda=\lambda$ et $\Delta^{k} \lambda \neq \lambda$ pour $k=1, \ldots, p-1$, où $\Delta x=2 x \bmod 1)$. La famille $\left\{\sigma_{1}, \ldots, \sigma_{p}\right\}$ vérifiant la relation ci-dessus est unique, et l'on note

$$
\mathcal{C}_{\lambda}=\left\{\sigma_{k} \cdots \sigma_{1} \lambda: k=1, \ldots, p\right\}
$$

Il est clair que les points périodiques d'ordre inférieur ou égal à un entier $m, m \in \mathbb{N}^{*}$, sont de la forme $k /\left(2^{p}-1\right)$, où $p \in\{1, \ldots, m\}$ et $k \in\left\{0,1, \ldots, 2^{p}-1\right\}$. La condition (4) est équivalente à la suivante: pour tout $\lambda \in] 0,1\left[, p\right.$-périodique, $2 \leq p \leq Q$, il existe $y \in \mathcal{C}_{\lambda}$, tel que

$$
H_{0}(y) H_{1}(y) \neq 0 \text {. }
$$


Pour $\lambda \in[0,1]$, on note $A_{\lambda}=\left\{\sigma_{n} \cdots \sigma_{1} \lambda: n \geq 1, \sigma_{1}, \ldots, \sigma_{n} \in\left\{S_{0}, S_{1}\right\}\right\}$. Nous aurons besoin des propriétés suivantes démontrées dans [4]:

Soit $\lambda \in[0,1]$. Si $\lambda$ est périodique, alors un (et uniquement un) des points $S_{0} \lambda$ et $S_{1} \lambda$ est périodique. Si $\lambda$ n'est pas périodique, les points de $A_{\lambda}$ sont distincts deux à deux et ne sont pas périodiques.

Preuve Du Lemme 2.2. On note $Z$ l'ensemble des zéros de $u_{0} u_{1}$, et $|E|$ le cardinal d'un ensemble quelconque $E$. Rappelons que $|Z|=Q$. Les opérateurs $P_{i}$ étant positifs et tels que $P_{i} 1=1$, il suffit de prouver qu'il existe $\delta>0$ tel que l'on ait, pour tous $\lambda \in[0,1], \omega_{1}, \ldots, \omega_{r} \in\{0,1\}$

$$
P_{1} P_{\omega_{r}} \cdots P_{\omega_{1}} h(\lambda) \geq \delta
$$

a) Soit $r_{1}=\left[\log _{2}(2 N+1)\right]+1$. Si $\lambda$ est non périodique et tel que $A_{\lambda} \cap Z=\varnothing$, alors $\Pi_{m}^{\omega} h(\lambda)>0$, pour tous $m \geq r_{1}, \omega \in \Omega$. En effet, sinon on aurait $h\left(\sigma_{m} \cdots \sigma_{1} \lambda\right)=0$ pour chaque $\sigma_{m}, \ldots, \sigma_{1} \in\left\{S_{0}, S_{1}\right\}$, ce qui est impossible car $h$ admet au plus $2 N+1$ racines.

b) Soit $r_{2}=r_{1}+Q$. Si $\lambda$ est non périodique, alors $\Pi_{m}^{\omega} h(\lambda)>$ 0 , pour tous $m \geq r_{2}, \omega \in \Omega$. Pour prouver b), considérons les ensembles

$$
\begin{gathered}
A_{\lambda}^{Q}=\left\{\sigma_{k} \cdots \sigma_{1} \lambda: 1 \leq k \leq Q, \sigma_{i} \in\left\{S_{0}, S_{1}\right\}\right\}, \\
F_{\lambda}^{Q}=\left\{\sigma_{Q} \cdots \sigma_{1} \lambda: \sigma_{i} \in\left\{S_{0}, S_{1}\right\}\right\} .
\end{gathered}
$$

On note $p=\left|A_{\lambda}^{Q} \cap Z\right|$. Soit $\omega^{\prime} \in \Omega$ quelconque. On a $0 \leq p \leq Q$, et $\left|F_{\lambda}^{Q}-T_{Q, \lambda}^{\omega^{\prime}}\right| \leq 2^{Q-1}+2^{Q-2}+\cdots+2^{Q-p}=2^{Q}-2^{Q-p}$. Pour prouver cette inégalité, on peut par exemple représenter l'ensemble $A_{\lambda}^{Q}$ sous la forme d'un arbre dyadique de racine $\lambda$ (admettant pour fils $\lambda / 2$ et $\lambda / 2+1 / 2$ ... etc ...), et remarquer que le nombre $\left|F_{\lambda}^{Q}-T_{Q, \lambda}^{\omega^{\prime}}\right|$ est d'autant plus grand que les éléments de $Z$ sont proches de $\lambda$ dans l'arbre. Il en résulte que $\left|T_{Q, \lambda}^{\omega^{\prime}}\right| \geq 2^{Q-p}>Q-p$. Les points de $A_{\lambda}$ étant distincts deux à deux, on en déduit qu'il existe $y \in T_{Q, \lambda}^{\omega^{\prime}}$ tel que $A_{y} \cap Z=\varnothing$. Rappelons que $y$ est nécessairement non périodique. Soient maintenant $m \geq 1$ et $\omega \in \Omega$ quelconques: il existe $y_{m} \in T_{Q, \lambda}^{\theta^{m} \omega}$ non périodique tel que $A_{y_{m}} \cap Z=\varnothing$. Du a), il vient que $\Pi_{m}^{\omega} h\left(y_{m}\right)>0$ pour tout $m \geq r_{1}$, d'où, d'après le Lemme $2.1, \Pi_{m+Q}^{\omega} h(\lambda)>0$, ce qui prouve b).

c) Soit $r=r_{2}+Q$. Si $\lambda$ est périodique, $\lambda \neq 0$, alors $\Pi_{m}^{\omega} h(\lambda)>$ 0 , pour tous $m \geq r, \omega \in \Omega$. Démontrons tout d'abord que, pour tout 
$\omega^{\prime} \in \Omega$, il existe un élément $y$ de $T_{Q, \lambda}^{\omega^{\prime}}$ non périodique: dans le cas contraire, en vertu des propriétés sur les points périodiques rappelées ci-dessus, l'ensemble $T_{Q, \lambda}^{\omega^{\prime}}$ serait en fait réduit à un seul élément qui en outre appartiendrait à $\mathcal{C}_{\lambda}$, d'où $u_{0}(t) u_{1}(t)=0$ pour tout $t \in \mathcal{C}_{\lambda}$, ce qui contredit l'hypothèse (7).

Soit $m \in \mathbb{N}^{*}$. Il existe donc $y \in T_{Q, \lambda}^{\theta^{m} \omega}$ non périodique, de sorte qu'on a, pour tout $m \geq r_{2}, \Pi_{m}^{\omega} h(y)>0$ et donc d'après le Lemme 2.1, $\Pi_{m+Q}^{\omega} h(\lambda)>0$. Le point c) est prouvé.

On a en particulier démontré que, pour tout $\omega_{1}, \ldots, \omega_{r}, \omega_{r+1} \in$ $\{0,1\}$ et tout $\lambda \neq 0, P_{\omega_{r+1}} P_{\omega_{r}} \cdots P_{\omega_{1}} h(\lambda)>0$. Remarquons que, si $\omega_{r+1}=\omega_{r}=\cdots=\omega_{2}=0$ et $\omega_{1}=1$, alors $\Pi_{r+1}^{\omega} h(0)=u_{0}(0) \cdots u_{0}(0)$ $u_{1}(1 / 2) h(1 / 2)=h(1 / 2)$, ce dernier terme pouvant être nul. Pour $\lambda=0$, il est donc nécessaire d'avoir $\omega_{r+1}=1$.

d) $($ cas $\lambda=0)$. On a $P_{1} P_{\omega_{r}} \cdots P_{\omega_{1}} h(0)>0$, pour tous $\omega_{1}, \ldots, \omega_{r} \in$ $\{0,1\}$. En effet, comme $u_{1}(0)=0$, on obtient

$$
\begin{aligned}
& P_{1} P_{\omega_{r}} \cdots P_{\omega_{1}} h(0) \\
& \quad=\sum_{\sigma_{2}, \ldots, \sigma_{r+1} \in\left\{S_{0}, S_{1}\right\}} u_{1}\left(\frac{1}{2}\right) u_{\omega_{r}}\left(\sigma_{2} \frac{1}{2}\right) \cdots u_{\omega_{1}}\left(\sigma_{r+1} \cdots \sigma_{2} \frac{1}{2}\right) h\left(\sigma_{r+1} \cdots \sigma_{2} \frac{1}{2}\right) \\
& \quad=P_{\omega_{r}} \cdots P_{\omega_{1}} h\left(\frac{1}{2}\right)
\end{aligned}
$$

ce dernier terme étant positif d'après ce qui précède.

Notons que $r$ est bien indépendant de la fonction $h$. Nous pouvons maintenant prouver (8). Soient $\omega_{1}, \ldots, \omega_{r} \in\{0,1\}$. Il existe une constante $\delta_{\left(\omega_{1}, \ldots, \omega_{r}\right)}>0$ ne dépendant que de $\left(\omega_{1}, \ldots, \omega_{r}\right)$ telle que $P_{1} P_{\omega_{r}} \cdots P_{\omega_{1}} h \geq \delta_{\left(\omega_{1}, \ldots, \omega_{r}\right)}$. On en déduit (8) avec $\delta=\min \left\{\delta_{\left(\omega_{1}, \ldots, \omega_{r}\right)}\right.$ : $\left.\omega_{i} \in\{0,1\}\right\}$.

\section{DÉmonstration du ThÉorème 1.2. Soit $\omega \in \Omega$.}

$1^{\text {er }}$ cas: il existe $k \in \mathbb{N}^{*}$ tel que $\omega_{n}=0$ pour tout $n>k$. Alors $\Pi_{n}^{\omega}=P_{0}^{n-k} \Pi_{k}^{\omega}$. On déduit de l'étude des itérées de $P_{0}$ faite dans [2] que, pour tout $f \in E$, la suite $\left\{\Pi_{n}^{\omega} f: n \geq 1\right\}$ converge uniformément vers la constante $\Pi_{k}^{\omega} f(0)$.

$2^{\text {ieme }}$ cas: il existe une suite strictement croissante $\{\phi(n)\}_{n \geq 1}$ d'entiers positifs tels que $\omega_{\phi(n)}=1$. Commençons par supposer que

- $f \in \mathcal{T}_{N}$ : on peut choisir les $\phi(n)$ tels que $\phi(n+1)-\phi(n)>r+1$, où $r$ est l'entier défini dans le Lemme 2.2. Soit $\psi(n)=\phi(n)-r-1$. 
La famille $\left\{\Pi_{\psi(n)}^{\omega} f: n \geq 1\right\}$ est bornée dans l'espace $\mathcal{T}_{N}$ qui est de dimension finie. On peut donc en extraire une sous-suite $\left\{\Pi_{\mathcal{\tau}(n)}^{\omega} f: n \geq\right.$ 1) convergeant uniformément vers une fonction $g$ de $\mathcal{T}_{N}$.

Nous allons démontrer que $g$ est identiquement égale à

$$
c=\inf _{\lambda \in[0,1]}[g(\lambda)] .
$$

A cet effet procédons par l'absurde et supposons qu'il existe $\lambda \in[0,1]$ tel que $g(\lambda)>c$. On a $\Pi_{\tau(n+1)}^{\omega}=R_{n} \Pi_{\tau(n)}^{\omega}$ où

$$
R_{n}=P_{\omega_{\tau(n+1)}} \cdots P_{\omega_{\tau(n)+r+2}} P_{\omega_{\tau(n)+r+1}} P_{\omega_{\tau(n)+r}} \cdots P_{\omega_{\tau(n)+1}} .
$$

Rappelons que par construction $\omega_{\tau(n)+r+1}=1$. Le Lemme 2.2 appliqué avec $h=g-c$ assure l'existence d'une constante $\delta>0$ telle que l'on ait $R_{n}(g-c) \geq \delta$, ou encore $R_{n} g \geq c+\delta$, pour tout $n \geq 1$. Or on a $\Pi_{\tau(n+1)}^{\omega} f-R_{n} g=R_{n}\left(\Pi_{\tau(n)}^{\omega} f-g\right)$, d'où $\left\|\Pi_{\tau(n+1)}^{\omega} f-R_{n} g\right\|_{\infty} \leq$ $\left\|\Pi_{\tau(n)}^{\omega} f-g\right\|_{\infty}$. La suite $\left\{R_{n} g: n \geq 1\right\}$ converge donc uniformément vers $g$. Il en résulte que $\lim _{n \rightarrow+\infty}\left(\inf _{\lambda \in[0,1]} R_{n} g(\lambda)\right)=c$, ce qui est impossible d'après l'inégalité ci-dessus. Donc $g=c$. On conclut que $\left\{\Pi_{n}^{\omega} f: n \geq 1\right\}$ converge uniformément vers $c$ en remarquant que, pour tout $m \geq \tau(n)$,

$$
\left\|\Pi_{m}^{\omega} f-c\right\|_{\infty}=\left\|P_{\omega_{m}} \cdots P_{\omega_{\tau(n)+1}}\left(\Pi_{\tau(n)}^{\omega} f-c\right)\right\|_{\infty} \leq\left\|\Pi_{\tau(n)}^{\omega} f-c\right\|_{\infty} .
$$

Passons au cas général

- $f \in E$ : il existe une suite $\left\{f_{k}\right\}_{k \geq 1}$ de polynômes trigonométriques convergeant dans $E$ vers $f$. Soit $\varepsilon>0$. On a

$$
\begin{aligned}
\left\|\Pi_{q}^{\omega} f-\Pi_{p}^{\omega} f\right\|_{\infty} \leq & \left\|\Pi_{q}^{\omega} f-\Pi_{q}^{\omega} f_{k}\right\|_{\infty}+\left\|\Pi_{q}^{\omega} f_{k}-\Pi_{p}^{\omega} f_{k}\right\|_{\infty} \\
& +\left\|\Pi_{p}^{\omega} f_{k}-\Pi_{p}^{\omega} f\right\|_{\infty} \\
\leq & 2\left\|f_{k}-f\right\|_{\infty}+\left\|\Pi_{q}^{\omega} f_{k}-\Pi_{p}^{\omega} f_{k}\right\|_{\infty} .
\end{aligned}
$$

On fixe $k$ assez grand pour que $\left\|f_{k}-f\right\|_{\infty} \leq \varepsilon / 3$. On sait que, pour $\ell$ assez grand, $\Pi_{\ell}^{\omega} f_{k} \in \mathcal{T}_{N}$ (car $P_{0}$ et $P_{1}$ contractent les degrés). On déduit de ce qui précède que la suite $\left\{\Pi_{n}^{\omega} f_{k}: n \geq 1\right\}$ converge dans $E$, et finalement que $\left\{\Pi_{n}^{\omega} f: n \geq 1\right\}$ est une suite de Cauchy dans $E$. Cette dernière suite converge donc vers une fonction $h \in E$, et on a en particulier $\lim _{n \rightarrow+\infty} P_{1}\left(P_{\omega_{\phi(n)-1}} \cdots P_{\omega_{1}} f\right)=P_{1} h=h\left(\operatorname{car} \omega_{\phi(n)}=1\right)$. On conclut en utilisant le fait que, sous l'hypothèse (7) (qui assure que 
$u_{1}$ n'a pas de cycle périodique invariant), les fonctions $P_{1}$-invariantes sont constantes, voir [2].

\section{Démonstration du Théorème 1.3.}

Nous conservons les notations et hypothèses précédentes (voir début du Paragraphe 2), et nous nous proposons de démontrer le Théorème 1.3, c'est-à-dire l'inégalité (6) pour tout $f \in E^{\eta}$. Pour simplifier les notations, on suppose que $\eta=1$ (la démonstration est identique pour $\eta \in] 0,1]$ quelconque). Remarquons tout d'abord que $P_{0}, P_{1}$, et donc chaque $\Pi_{n}^{\omega}$, sont des opérateurs bornés sur $E^{1}$. Plus précisément, on démontre facilement l'existence de constantes $C, R>0$ telles que l'on ait, pour $j=0,1$ et tout $f \in E^{1}$,

$$
\begin{gathered}
m_{1}\left(P_{j} f\right) \leq 2^{-1} m_{1}(f)+C\|f\|_{\infty}, \\
\left\|P_{j} f\right\|_{1} \leq 2^{-1}\|f\|_{1}+R\|f\|_{\infty} .
\end{gathered}
$$

On en déduit que, pour tout $\omega \in \Omega$,

$$
\left\|\Pi_{2}^{\omega} f\right\|_{1} \leq \frac{1}{4}\|f\|_{1}+\frac{3}{2} R\|f\|_{\infty},
$$

et plus généralement, pour tout $n \in \mathbb{N}^{*}$,

$$
\left\|\Pi_{n}^{\omega} f\right\|_{1} \leq 2^{-n}\|f\|_{1}+2 R\|f\|_{\infty}
$$

D'autre part, pour tout $f \in E$, les suites $\left\{P_{0}^{n} f: n \geq 1\right\}$ et $\left\{P_{1}^{n} f\right.$ : $n \geq 1\}$ convergent dans $E$. En vertu du Théorème de Ionescu-Tulcea et Marinescu [6], [9], la valeur propre 1 est l'unique valeur spectrale de module 1 pour $P_{0}$ et $P_{1}$, et on obtient les décompositions suivantes sur $E^{1}$ :

Pour $i=0,1$, il existe une mesure de probabilité $\nu_{i}, P_{i}$-invariante sur le tore, et un opérateur $Q_{i}$ borné sur $E^{1}$, de rayon spectral strictement inférieur à 1, tels que

$$
P_{i} f=\int f d \nu_{i}+Q_{i}(f), \quad \text { pour tout } f \in E^{1},
$$


avec, en outre, $Q_{i} \nu_{j}=0$ et $\nu_{i} \nu_{j}=\nu_{j}$ pour $i, j \in\{0,1\}$ (on a noté $\nu_{i}$ l'opérateur défini sur $E^{1}$ par $\left.\nu_{i}(f)=\int f d \nu_{i}\right)$. On en déduit que, pour tout $f \in E^{1}$,

$$
\begin{aligned}
\Pi_{n}^{\omega}(f)= & \nu_{\omega_{1}}(f)+\nu_{\omega_{2}} Q_{\omega_{1}} f+\cdots+\nu_{\omega_{n}} Q_{\omega_{n-1}} \cdots Q_{\omega_{1}} f \\
& +Q_{\omega_{n}} \cdots Q_{\omega_{1}} f .
\end{aligned}
$$

La démonstration du Théorème 1.3 utilise les trois lemmes suivants:

Lemme 3.1. Il existe une constante $D>0$ telle que pour tous $f \in$ $E^{1}, n \in \mathbb{N}^{*}, \omega_{1}, \ldots, \omega_{n} \in\{0,1\}$,

$$
\left\|Q_{\omega_{n}} \cdots Q_{\omega_{1}} f\right\|_{1} \leq D\|f\|_{1} \text {. }
$$

Lemme 3.2. On a pour tout $f \in E^{1}$ et tout $\omega=\left(\omega_{1}, \omega_{2}, \ldots, \omega_{n}, \ldots\right) \in \Omega$

a) $\lim _{n \rightarrow+\infty}\left\|Q_{\omega_{n}} \cdots Q_{\omega_{1}} f\right\|_{\infty}=0$.

b) $\lim _{n \rightarrow+\infty}\left\|Q_{\omega_{n}} \cdots Q_{\omega_{1}} f\right\|_{1}=0$.

Lemme 3.3. Pour tout réel $\beta>0$, il existe $M \in \mathbb{N}^{*}$ tel que pour tous $f \in E^{1}, n \geq M, \omega_{1}, \ldots, \omega_{n} \in\{0,1\}$,

$$
\left\|Q_{\omega_{n}} \cdots Q_{\omega_{1}} f\right\|_{1} \leq \beta\|f\|_{1} \text {. }
$$

Commençons par admettre ces lemmes, et donnons la

DÉmonstration Du ThÉorème 1.3. Soient $\beta$ tel que $0<\beta<1$, et $M$ l'entier du Lemme 3.3 correspondant à $\beta$. Soient $\omega \in \Omega$, et $k \in \mathbb{N}^{*}$ qu'on écrit sous la forme $k=q M+r$, où $q \in \mathbb{N}$ et $r \in\{0, \ldots, M-1\}$. Alors

$$
\begin{aligned}
\left\|Q_{\omega_{k}} \cdots Q_{\omega_{1}} f\right\|_{1} & \leq D \beta^{q}\|f\|_{1} \\
& =D^{\prime}\left(\beta^{1 / M}\right)^{k}\|f\|_{1} .
\end{aligned}
$$

On pose $\rho=\beta^{1 / M}$ et $T_{k} f=Q_{\omega_{k}} \cdots Q_{\omega_{1}} f$. Utilisant (13) et le fait que, pour $g \in E^{1}$ et $j=0,1,\left\|\nu_{j}(g)\right\|_{1}=\left|\nu_{j}(g)\right| \leq\|g\|_{\infty} \leq\|g\|_{1}$, on montre aisément que

$$
\begin{aligned}
\left\|\Pi_{n+p}^{\omega} f-\Pi_{n}^{\omega} f\right\|_{1} & \leq\left\|T_{n} f\right\|_{1}+\sum_{k=n}^{n+p}\left\|T_{k} f\right\|_{1} \\
& \leq 2 D^{\prime}\left(\sum_{k=n}^{n+p} \rho^{k}\right)\|f\|_{1} .
\end{aligned}
$$


On conclut grâce au critère de Cauchy, et au Théorème 1.2 qui permet d'identifier la limite à une constante.

Preuve Du Lemme 3.1. La démonstration de ce lemme est donnée dans [2]. Nous la reprenons ici car elle met en jeu une inégalité importante dont nous aurons besoin dans la suite. Grâce à (9), (12), et enfin aux relations entre $Q_{i}$ et $\nu_{j}$, on obtient les majorations suivantes:

$$
\begin{aligned}
\left\|Q_{\omega_{n}} \cdots Q_{\omega_{1}} f\right\|_{1} & =\left\|Q_{\omega_{n}} P_{\omega_{n-1}} \cdots P_{\omega_{1}} f\right\|_{1} \\
& =\left\|Q_{\omega_{n}} P_{\omega_{n-1}} \cdots P_{\omega_{1}} f\right\|_{\infty}+m_{1}\left(P_{\omega_{n}} \cdots P_{\omega_{1}} f\right) \\
& \leq 2\|f\|_{\infty}+2^{-1} m_{1}\left(P_{\omega_{n-1}} \cdots P_{\omega_{1}} f\right)+C\|f\|_{\infty},
\end{aligned}
$$

et finalement

$$
\begin{aligned}
\left\|Q_{\omega_{n}} \cdots Q_{\omega_{1}} f\right\|_{1} \leq & \left(2+C+C 2^{-1}+\cdots+C 2^{-(n-1)}\right)\|f\|_{\infty} \\
& +2^{-n} m_{1}(f) .
\end{aligned}
$$

Preuve Du Lemme 3.2. a) On obtient grâce à (13)

$$
\nu_{\omega_{n+1}} \Pi_{n}^{\omega} f=\sum_{i=1}^{n+1} \nu_{\omega_{i}} Q_{\omega_{i-1}} \cdots Q_{\omega_{1}} f .
$$

Rappelons que $\left\{\Pi_{n}^{\omega} f\right\}_{n \geq 1}$ converge uniformément vers une constante $c(f, \omega)$. On a en outre

$$
\begin{aligned}
\left\|\nu_{\omega_{n+1}}\left(\Pi_{n}^{\omega} f\right)-c(f, \omega)\right\|_{\infty} & =\left\|\nu_{\omega_{n+1}}\left[\Pi_{n}^{\omega} f-c(f, \omega)\right]\right\|_{\infty} \\
& \leq\left\|\Pi_{n}^{\omega} f-c(f, \omega)\right\|_{\infty},
\end{aligned}
$$

d'où, d'après le Théorème $1.2, \lim _{n \rightarrow+\infty}\left\|\nu_{\omega_{n+1}}\left(\Pi_{n}^{\omega} f\right)-c(f, \omega)\right\|_{\infty}=0$. On en déduit que $\left\{\sum_{i=1}^{n} \nu_{\omega_{i}} Q_{\omega_{i-1}} \cdots Q_{\omega_{1}} f: n \geq 1\right\}$ converge dans $E$ vers $c(f, \omega)$ quand $n \rightarrow+\infty$. On utilise à nouveau (13) pour en déduire le a) du lemme.

b) On pose $a_{n}=m_{1}\left(Q_{\omega_{n}} \cdots Q_{\omega_{1}} f\right)$ et $b_{n}=\left\|Q_{\omega_{n}} \cdots Q_{\omega_{1}} f\right\|_{\infty}$. En vertu du a), il reste à prouver que $\lim _{n \rightarrow+\infty} a_{n}=0$. Or, on a $a_{k}=m_{1}\left(P_{\omega_{k}} Q_{\omega_{k-1}} \cdots Q_{\omega_{1}} f\right)$, d'où d'après $(9), a_{k} \leq 2^{-1} a_{k-1}+C b_{k-1}$, et pour tout $p \geq 1$,

$$
a_{n+p} \leq 2^{-p} a_{n}+C\left(b_{n+p-1}+2^{-1} b_{n+p-2}+\cdots+2^{-p+1} b_{n}\right) .
$$

L'assertion b) résulte donc du point a) et du fait que la suite $\left\{a_{n}\right\}_{n \geq 1}$ est bornée ( $c f$. Lemme 3.1). 
Preuve Du Lemme 3.3. On note $\mathcal{S}_{1}$ la sphère unité de $E^{1}$. Par ailleurs on définit sur $\Omega$ la distance $d\left(\omega, \omega^{\prime}\right)=\sum_{k \geq 1} 2^{-k}\left|\omega_{k}-\omega_{k}^{\prime}\right|$. Rappelons que $(\Omega, d)$ est compact.

Nous procédons par l'absurde en supposant qu'il existe un réel $\beta>0$ pour lequel on a la propriété suivante: pour tout $n \in \mathbb{N}^{*}$, il existe $\psi(n) \geq n, \omega_{1}^{\psi(n)}, \ldots, \omega_{\psi(n)}^{\psi(n)} \in\{0,1\}, f_{\psi(n)} \in \mathcal{S}_{1}$, tels que

$$
\left\|Q_{\omega_{\psi(n)}^{\psi(n)}} \cdots Q_{\omega_{1}^{\psi(n)}} f_{\psi(n)}\right\|_{1}>\beta .
$$

On pose $\omega^{\psi(n)}=\left(\omega_{1}^{\psi(n)}, \ldots, \omega_{\psi(n)}^{\psi(n)}, 0,0, \ldots\right) \in \Omega$. En vertu du Théorème d'Ascoli et de la compacité de $\Omega$, il existe une suite d'entiers positifs $\{\phi(n)\}_{n \geq 1}$ strictement croissante (extraite de $\{\psi(n)\}_{n \geq 1}$ ) telle que $\left\{\omega^{\phi(n)}\right\}_{n \geq 1}$ converge vers $\omega \in \Omega$, et telle que $\left\{f_{\phi(n)}\right\}_{n \geq 1}$ converge dans $E$ vers $f \in E^{1}$, avec $\|f\|_{1} \leq 1$. Posant

$$
A_{n}=Q_{\omega_{\phi(n)}^{\phi(n)}} \cdots Q_{\omega_{1}^{\phi(n)}},
$$

on obtient

$$
\beta<\left\|A_{n} f_{\phi(n)}\right\|_{1} \leq\left\|A_{n}\left(f_{\phi(n)}-f\right)\right\|_{1}+\left\|A_{n} f\right\|_{1} .
$$

Nous allons démontrer que ces deux derniers termes ont une limite nulle quand $n \rightarrow+\infty$, ce qui constituera bien une contradiction:

De (14), il vient que

$$
\left\|A_{n}\left(f_{\phi(n)}-f\right)\right\|_{1} \leq E\left\|f_{\phi(n)}-f\right\|_{\infty}+2^{-\phi(n)} m_{1}\left(f_{\phi(n)}-f\right),
$$

avec $E=2(C+1)$. En outre, on a $m_{1}\left(f_{\phi(n)}-f\right) \leq 2$, d'où

$$
\lim _{n \rightarrow+\infty}\left\|A_{n}\left(f_{\phi(n)}-f\right)\right\|_{1}=0 .
$$

La convergence de $\left\{\omega^{\phi(n)}\right\}_{n \geq 1}$ vers $\omega=\left(\omega_{1}, \ldots, \omega_{n}, \ldots\right)$ entraîne qu'il existe une suite $\{k(n)\}_{n \geq 1}$ d'entiers positifs, avec $\lim _{n \rightarrow+\infty} k(n)=$ $+\infty$, telle que $w_{k}^{\phi(n)}=\omega_{k}$ pour tout $1 \leq k \leq k(n)$. Si $k(n) \geq \phi(n)$, alors $A_{n}=Q_{\omega_{\phi(n)}} \cdots Q_{\omega_{1}}$. Si $k(n)<\phi(n)$, alors

$$
A_{n}=Q_{\omega_{\phi(n)}^{\phi(n)}} \cdots Q_{\omega_{k(n)+1}^{\phi(n)}}\left(Q_{\omega_{k(n)}} \cdots Q_{\omega_{1}}\right)
$$

d'où $\left\|A_{n} f\right\|_{1} \leq D\left\|Q_{\omega_{k(n)}} \cdots Q_{\omega_{1}} f\right\|_{1}$ d'après le Lemme 3.1. On déduit du Lemme 3.2.b) que $\lim _{n \rightarrow+\infty}\left\|A_{n} f\right\|_{1}=0$, ce qui achève la démonstration du Lemme 3.3. 


\section{References.}

[1] Coifman, R., Meyer, Y., Quake, S., Wickerhauser M.V., Signal processing and compression with wave packets. Proc. of the Conference on Wavelets, Marseille, Spring 1989.

[2] Conze, J. P., Raugi, A., Fonctions harmoniques pour un opérateur de transition et applications. Bull. Soc. Math. France 118 (1990), 273-310.

[3] Esteban, D., Galand, C., Application of Quadrature Mirror Filters to Split-band Voice Coding Schemes. Proc. of ICASSP, Hartford, Connecticut, 1977.

[4] Hervé, L., Etude d'opérateurs quasi-compacts et positifs. Applications aux opérateurs de transfert Ann. Inst. H. Poincaré, Probabilités et Statistique. 30, (1994), 437-466.

[5] Hervé, L., Comportement asymptotique dans l'algorithme de transformée en ondelettes. Lien avec la régularité de l'ondelette. Revista Mat. Iberoamericana 11 (1995), 431-451.

[6] Ionescu-Tulcéa, C.T., Marinescu, G., Théorie ergodique pour une classe d'opérations non complétement continues. Ann. of Math. 52 (1950), 140-147.

[7] Keane, M., Strongly mixing g-measures. Invent. Math. 16 (1972), 309-324.

[8] Mallat, S., Multiresolution approximations and wavelet orthonormal bases of $L^{2}(\mathbb{R})$. Trans. Amer. Math. Soc. 315 (1989), 69-88.

[9] Norman, M. F., Markov processes and learning models. Academic Press, 1972.

Recibido: 3 de mayo de 1.995

Revisado: 6 de septiembre de 1.995

Loïc Hervé

I.R.M.A.R.-Universitè de Rennes 1

Laboratoire de Probabilités

Campus de Beaulieu, 35042 Rennes Cedex

FRANCE

herve@univ-rennes1.fr 